\title{
Supporting Information: Photoinduced Carrier Dynamics at the Interface of Black Phosphorus and Bismuth Vanadate
}

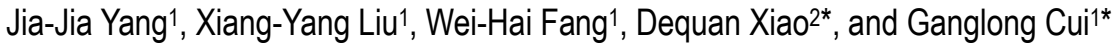 \\ ${ }^{1}$ Key Laboratory of Theoretical and Computational Photochemistry, Ministry of Education, College of Chemistry, \\ Beijing Normal University, Beijing 100875, China and 2Center for Integrative Materials Discovery, Department \\ of Chemistry and Chemical Engineering, University of New Haven, 300 Boston Post Road, West Haven, \\ Connecticut 06516, United States
}

Email: dxiao@newhaven.edu; ganglong.cui@bnu.edu.cn

I、Tables S2

Table S1 Energies of states of BP and BVO in BP/BVO at the optimized structure calculated by the HSE06 method. S2

Table S2 Energies of states of BP and BVO in BP/BVO at the optimized structure and a MD snapshot calculated by the PBE method

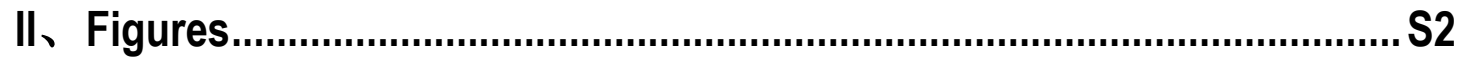

Fig. S1: DOS of BP/BVO calculated by the HSE06 method. S2

Fig. S2: Time-dependent state populations of state-388 and state-389 in the interfacial hole transfer dynamics simulations. S3

Fig. S3: Overlap of ground- (blue) and excited-state (orange) structures. S4

III、Cartesian Coordinates S5 


\section{I、Tables}

Table S1: Energies of States of BP and BVO in BP/BVO at the Optimized Structure (i.e. $0 \mathrm{~K}$ ) Calculated by the HSE06 Method.

\begin{tabular}{|c|c|c|c|c|c|c|c|c|c|}
\hline BP & $\begin{array}{c}1.85 \\
(432)\end{array}$ & $\begin{array}{c}1.83 \\
(431)\end{array}$ & $\begin{array}{c}1.78 \\
(429)\end{array}$ & $\begin{array}{c}1.63 \\
(423)\end{array}$ & $\begin{array}{c}1.46 \\
(\mathrm{CBM})\end{array}$ & $\begin{array}{c}-0.50 \\
(\mathrm{VBM})\end{array}$ & $\begin{array}{c}-1.28 \\
(395)\end{array}$ & $\begin{array}{c}-1.35 \\
(394)\end{array}$ & $\begin{array}{c}-1.76 \\
(393)\end{array}$ \\
\hline \multirow{2}{*}{ BVO } & 0.78 & 0.77 & 0.57 & 0.56 & 0.51 & -2.98 & -3.06 & -3.12 & -3.15 \\
& $(401)$ & $(400)$ & $(399)$ & $(398)$ & $(\mathrm{CBM})$ & $(\mathrm{VBM})$ & $(381)$ & $(380)$ & $(378)$ \\
\hline
\end{tabular}

Table S2: Energies of States of BP and BVO in BP/BVO at the Optimized Structure (top; i.e. 0 K) and at a MD Snapshot (bottom; i.e. $300 \mathrm{~K}$ ) Calculated by the PBE Method.

\begin{tabular}{|c|c|c|c|c|c|c|c|c|c|}
\hline BP & $\begin{array}{c}1.16 \\
(\mathrm{CBM})\end{array}$ & $\begin{array}{c}-0.13 \\
(\mathrm{VBM})\end{array}$ & $\begin{array}{c}-0.86 \\
(395)\end{array}$ & $\begin{array}{c}-0.93 \\
(394)\end{array}$ & $\begin{array}{c}-1.29 \\
(393)\end{array}$ & $\begin{array}{c}-1.32 \\
(392)\end{array}$ & $\begin{array}{c}-1.46 \\
(389)\end{array}$ & $\begin{array}{c}-1.97 \\
(388)\end{array}$ & $\begin{array}{c}-2.21 \\
(383)\end{array}$ \\
\hline \multirow{2}{*}{ BVO } & 1.12 & 0.99 & 0.76 & 0.65 & 0.41 & 0.18 & 0.17 & 0.14 & -2.23 \\
& $(420)$ & $(415)$ & $(410)$ & $(405)$ & $(400)$ & $(399)$ & $(398)$ & $(\mathrm{CBM})$ & $(\mathrm{VBM})$ \\
\hline
\end{tabular}

\begin{tabular}{|c|c|c|c|c|c|c|c|c|c|}
\hline BP & $\begin{array}{c}1.10 \\
(\mathrm{CBM})\end{array}$ & $\begin{array}{c}-0.09 \\
(\mathrm{VBM})\end{array}$ & $\begin{array}{c}-0.73 \\
(395)\end{array}$ & $\begin{array}{c}-1.10 \\
(393)\end{array}$ & $\begin{array}{c}-1.24 \\
(391)\end{array}$ & $\begin{array}{c}-1.46 \\
(389)\end{array}$ & $\begin{array}{c}-1.86 \\
(387)\end{array}$ & $\begin{array}{c}-1.98 \\
(385)\end{array}$ & $\begin{array}{c}-2.00 \\
(383)\end{array}$ \\
\hline \multirow{2}{*}{ BVO } & 1.08 & 0.95 & 0.79 & 0.53 & 0.26 & 0.19 & 0.12 & 0.10 & -2.25 \\
& $(420)$ & $(415)$ & $(410)$ & $(405)$ & $(400)$ & $(399)$ & $(398)$ & $(\mathrm{CBM})$ & $(\mathrm{VBM})$ \\
\hline
\end{tabular}

\section{II、 Figures}

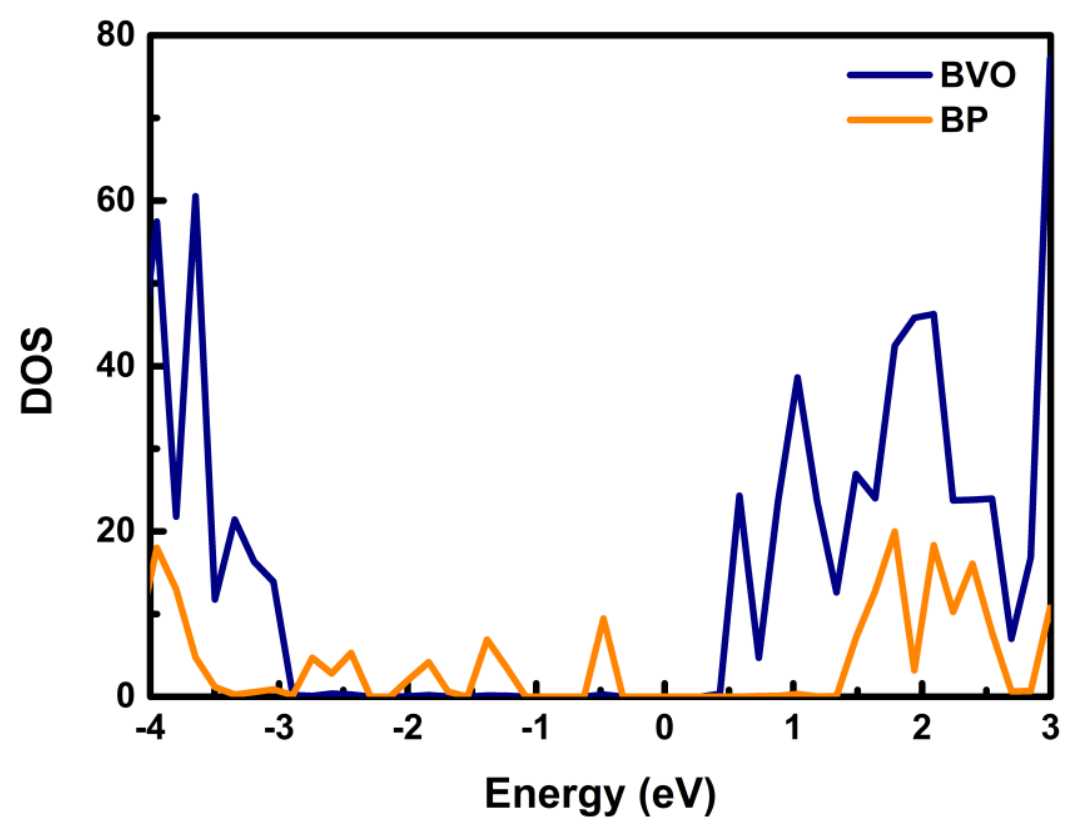

Fig. S1: DOS of BP/BVO at the optimized structure calculated by the HSE06 method. 

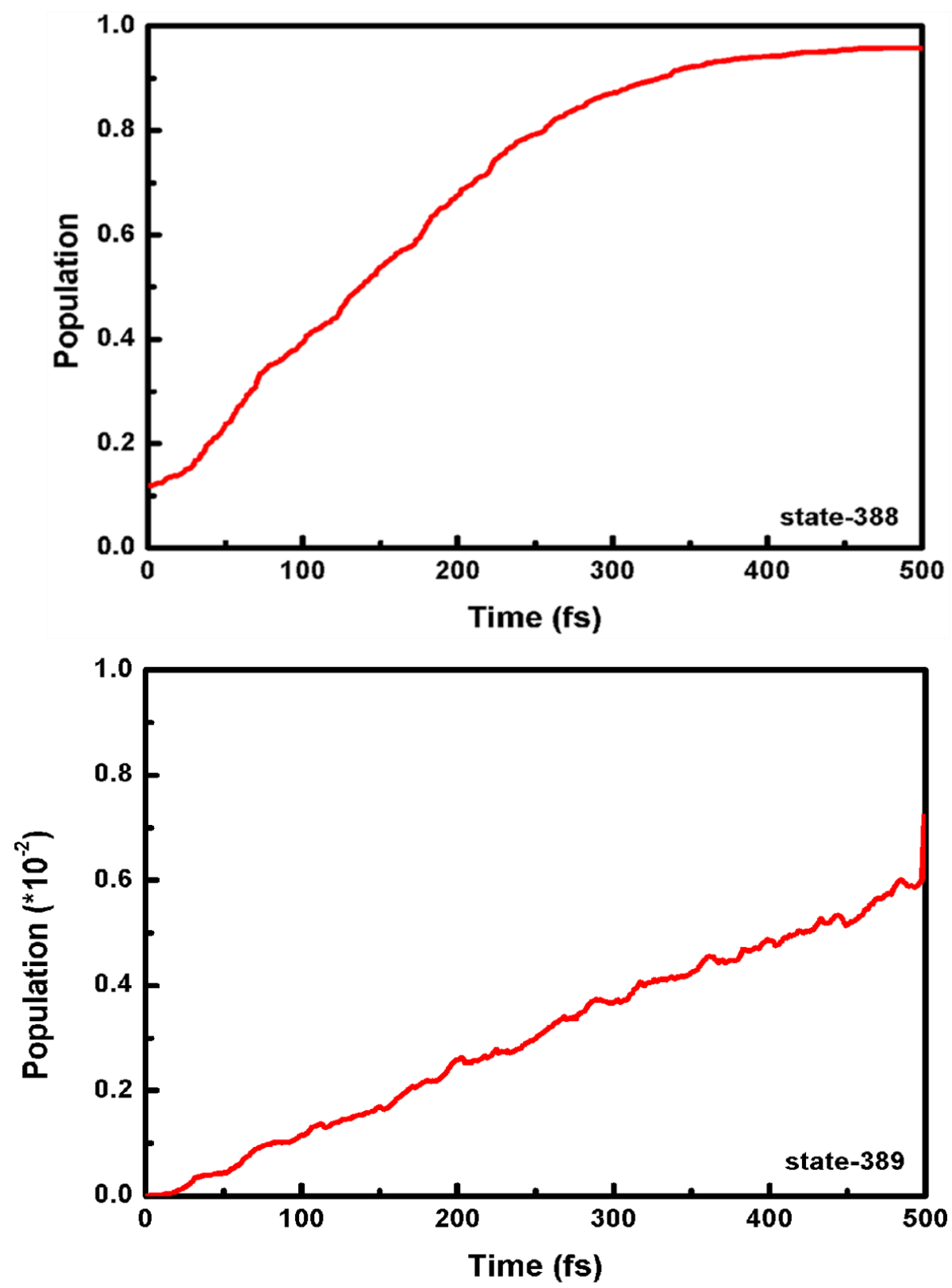

Fig. S2: Time-dependent state populations of state-388 and state-389 in the interfacial hole transfer dynamics simulations. See main text for details. 

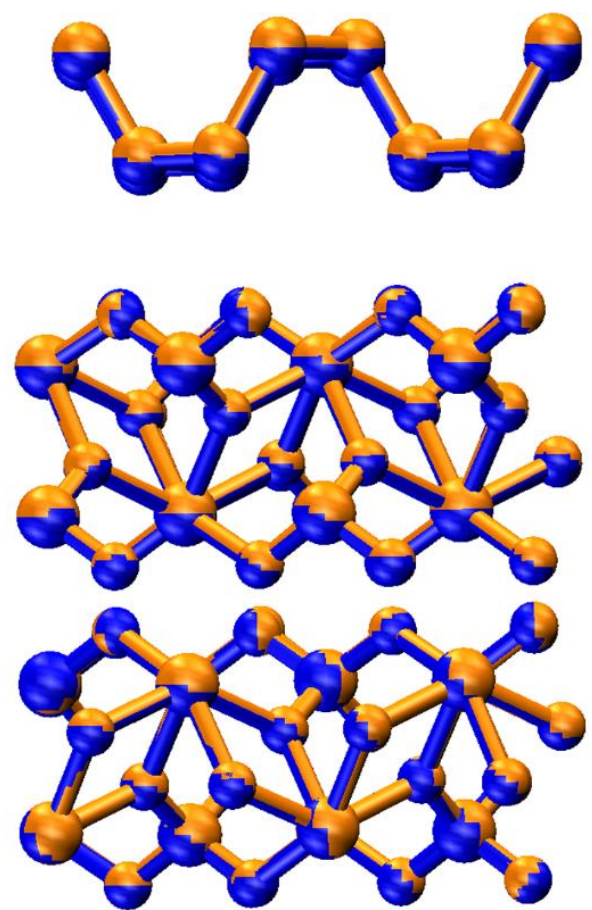

Fig. S3: Overlap of ground- (blue) and excited-state (orange) structures, in which excited-state one is optimized with the constrained DFT method implemented in CP2K. 


\section{III、Cartesian Coordinates}

120

$0 \mathrm{~K} \quad$ (xyz format, in angstrom)

0

0.7809407700

2.5433268898

17.2124614173

0

1.4385670358

2.0400930495

20.0335375598

O $\quad 3.4092626567$

0.0975189477

11.1425399638

3.9488329157

4.4713258058

14.0235204034

4.4434702981

5.0371848182

17.2169537178

0

3.8201330612

0.6000718707

20.0312820683

1.7813318439

2.5214212004

11.1472555350

1.2728064813

3.1658436443

14.0865027097

4.4283844521

2.5248223678

12.1769358077

3.8315317971

3.0866951081

9.2923144338

1.7893557609

5.0407510224

18.1940225494

1.2545807213

0.5962244643

15.3041208623

0.7500469153

0.0944695403

12.1780177014

1.4526342401

4.5416666011

9.2852691281

3.4291751749

2.6146062728

18.1850531440

1.9665343551

15.3078214502

2.5378169239

17.2189726847

2.0534574308

20.0342444181

0.0961317636

11.1349024164

4.4720171015

14.0880158283

5.0376834053

17.2121253428

0.5951241030

20.0321018834

2.5301164476

11.2005196402

3.1728302478

14.0282903752

2.5259303613

12.1855156868

3.0878055091

9.2915825846

5.0404097854

18.1944307694

0.5971764195

15.3091054917

0.0926142665

12.1886208947

4.5422641982

9.2965617411

2.6119861672

18.1824735784

1.9621937259

15.3089443023

7.6312499038

17.2155960640

7.0688311335

20.1007491415

5.1268244560

11.1445825016

9.5605142432

14.0886968760

10.0620298739

17.1501080676

5.6061577325

20.1013967628

7.6068323449

11.1549339433

8.1956057663

14.0245880062 


\begin{tabular}{|c|c|c|c|}
\hline 0 & 4.4602119765 & 7.6154496464 & 12.1790768038 \\
\hline 0 & 3.7977297789 & 8.1054881016 & 9.2900209995 \\
\hline 0 & 1.8957150881 & 10.0599551009 & 18.1921221788 \\
\hline 0 & 1.2903942813 & 5.6856183019 & 15.3104644266 \\
\hline 0 & 0.7889472921 & 5.1173616525 & 12.1841808931 \\
\hline 0 & 1.4835875166 & 9.6374220330 & 9.2873380347 \\
\hline 0 & 3.4633144101 & 7.6297770064 & 18.1931053667 \\
\hline 0 & 4.0275846561 & 6.9883532819 & 15.3082992554 \\
\hline 0 & 6.0364986798 & 7.6294837057 & 17.2200689441 \\
\hline 0 & 6.6944086948 & 7.0683229945 & 20.1084725087 \\
\hline 0 & 8.6461630015 & 5.1227548241 & 11.1443948938 \\
\hline 0 & 9.2114311169 & 9.5487603649 & 14.0252724220 \\
\hline 0 & 9.7092615853 & 10.0604377471 & 17.1539295161 \\
\hline 0 & 9.0124845414 & 5.6022865745 & 20.1071474569 \\
\hline 0 & 7.0319230627 & 7.6077335616 & 11.2126219356 \\
\hline 0 & 6.5343939882 & 8.1941583868 & 14.0252514462 \\
\hline 0 & 9.6867365880 & 7.5482362782 & 12.1797734921 \\
\hline 0 & 9.0235680260 & 8.1062162405 & 9.2962203238 \\
\hline 0 & 7.0517335987 & 10.0601980389 & 18.1899368125 \\
\hline 0 & 6.4506109592 & 5.6779014850 & 15.3098717091 \\
\hline 0 & 6.0074479902 & 5.1220807197 & 12.1783005035 \\
\hline 0 & 6.6391565098 & 9.5579314800 & 9.2906314326 \\
\hline 0 & 8.6901472077 & 7.6392088849 & 18.2504592500 \\
\hline 0 & 9.1907218779 & 6.9837580379 & 15.3125219594 \\
\hline $\mathrm{Bi}$ & -0.0077326475 & 1.3099108547 & 10.2901004224 \\
\hline $\mathrm{Bi}$ & 2.6169182390 & 3.8138128701 & 16.2997349032 \\
\hline $\mathrm{Bi}$ & 0.0347469342 & 3.8078005570 & 19.1119796219 \\
\hline $\mathrm{Bi}$ & 2.5979159244 & 1.3001529859 & 13.0564857872 \\
\hline $\mathrm{Bi}$ & 5.2101979807 & 1.3052231355 & 10.3093083776 \\
\hline $\mathrm{Bi}$ & 7.8347268908 & 3.8156110507 & 16.3048781108 \\
\hline $\mathrm{Bi}$ & 5.2287824592 & 3.8158273300 & 19.0813246537 \\
\hline $\mathrm{Bi}$ & 7.8058992663 & 1.3033619119 & 13.0850175622 \\
\hline $\mathrm{Bi}$ & 0.0344251694 & 6.3504244069 & 10.2841852879 \\
\hline $\mathrm{Bi}$ & 2.6574215905 & 8.8522643448 & 16.2793419301 \\
\hline $\mathrm{Bi}$ & 0.0865707913 & 8.8661840658 & 19.0837080472 \\
\hline $\mathrm{Bi}$ & 2.6264330116 & 6.3453694074 & 13.0515430334 \\
\hline $\mathrm{Bi}$ & 5.2370146639 & 6.3655923786 & 10.3176424965 \\
\hline $\mathrm{Bi}$ & 7.8611110578 & 8.8482466968 & 16.2924998247 \\
\hline $\mathrm{Bi}$ & 5.2641896465 & 8.8549287142 & 19.0637821548 \\
\hline $\mathrm{Bi}$ & 7.8465575706 & 6.3431892352 & 13.0735419578 \\
\hline V & 0.0128131466 & 1.2743863156 & 16.2499509223 \\
\hline V & 2.6324842633 & 3.8121501878 & 10.2199344896 \\
\hline V & 0.0294782720 & 3.8199909741 & 13.1278909781 \\
\hline V & 2.6186619383 & 1.2950978807 & 19.0903016163 \\
\hline
\end{tabular}




\begin{tabular}{|c|c|c|c|}
\hline V & 5.2229641357 & 1.2643022624 & 16.2533108781 \\
\hline V & 7.8009517963 & 3.8222485325 & 10.2331016747 \\
\hline V & 5.2208869595 & 3.8226147590 & 13.1000809484 \\
\hline V & 7.8039176760 & 1.2974162269 & 19.0744787593 \\
\hline V & 0.0487088337 & 6.3323677519 & 16.2738355962 \\
\hline V & 2.6505933407 & 8.8895006083 & 10.2266199087 \\
\hline V & 0.0670591776 & 8.8741332463 & 13.0828413542 \\
\hline V & 2.6290112355 & 6.3365932993 & 19.1300367933 \\
\hline V & 5.2392218211 & 6.3326024319 & 16.2877333848 \\
\hline V & 7.8438868470 & 8.8714359296 & 10.2293601213 \\
\hline V & 5.2757119597 & 8.8883735610 & 13.1121908707 \\
\hline V & 7.8503863318 & 6.3324939908 & 19.1448411904 \\
\hline$P$ & 4.4552038322 & 0.0558484908 & 25.1013464016 \\
\hline$P$ & 1.8618809063 & 1.6957119917 & 23.0691371752 \\
\hline$P$ & 0.7644317365 & 1.7551722819 & 25.0579779692 \\
\hline$P$ & 3.3536071956 & 0.0389728078 & 23.1197622653 \\
\hline$P$ & 9.6478379075 & 0.0553401516 & 25.1020728620 \\
\hline$P$ & 7.0556665463 & 1.6952732440 & 23.0650478059 \\
\hline$P$ & 5.9573990077 & 1.7551285654 & 25.0539731034 \\
\hline$P$ & 8.5470856390 & 0.0381343233 & 23.1197132919 \\
\hline$P$ & 4.4790007640 & 3.4443307488 & 24.9321223016 \\
\hline$P$ & 1.8988364682 & 5.0997255907 & 22.8951701132 \\
\hline$P$ & 0.7876300631 & 5.1002712824 & 24.8869909964 \\
\hline$P$ & 3.3639323334 & 3.3712872403 & 22.9488846145 \\
\hline$P$ & 9.6715897622 & 3.4445456611 & 24.9330681974 \\
\hline$P$ & 7.0932508247 & 5.1001572243 & 22.8961912655 \\
\hline$P$ & 5.9800641348 & 5.1004746847 & 24.8869021738 \\
\hline$P$ & 8.5579724821 & 3.3714246504 & 22.9490142268 \\
\hline$P$ & 4.4806799670 & 6.7607180990 & 24.9459206459 \\
\hline$P$ & 1.8977830947 & 8.5212275283 & 23.0624003274 \\
\hline$P$ & 0.7897071520 & 8.4366661055 & 25.0404397148 \\
\hline$P$ & 3.3768770466 & 6.8305517298 & 22.9536547890 \\
\hline$P$ & 9.6739159542 & 6.7607405704 & 24.9487872232 \\
\hline$P$ & 7.0909399891 & 8.5205925791 & 23.0634118710 \\
\hline$P$ & 5.9820438544 & 8.4363761762 & 25.0406588331 \\
\hline$P$ & 8.5707517245 & 6.8307532867 & 22.9569523912 \\
\hline
\end{tabular}

120

$300 \mathrm{~K} \quad$ (xyz format, in angstrom)

$\begin{array}{llll}0 & 0.6606968684 & 2.4706035246 & 17.2149884944 \\ 0 & 1.3762696152 & 1.7010053836 & 20.2299996131 \\ 0 & 3.6092081533 & -0.1695946569 & 11.0812016337 \\ 0 & 3.8563371784 & 4.5463083102 & 14.0234499786 \\ 0 & 4.3252071015 & 4.9493711951 & 17.2759040317\end{array}$




\begin{tabular}{|c|c|c|c|}
\hline 0 & 3.7774557099 & 0.4715237376 & 20.2504729018 \\
\hline 0 & 1.8828961536 & 2.5449597267 & 10.8926671485 \\
\hline 0 & 1.2760037153 & 3.1171273626 & 13.8408420095 \\
\hline 0 & 4.5094085039 & 2.4731345933 & 12.1409637015 \\
\hline 0 & 4.1479001026 & 3.3196670232 & 9.2853737030 \\
\hline 0 & 1.7886326336 & 5.0621076166 & 18.3913349209 \\
\hline 0 & 1.1824243094 & 0.6328621847 & 15.3534603604 \\
\hline 0 & 0.6943705846 & -0.1216073247 & 12.0174154389 \\
\hline 0 & 1.7790949859 & 4.7663539536 & 9.0361423148 \\
\hline 0 & 3.2702645713 & 2.4523729081 & 18.3112829065 \\
\hline 0 & 3.7101778332 & 1.8563967758 & 15.2975616506 \\
\hline 0 & 5.8208664138 & 2.5180839775 & 17.2051133493 \\
\hline 0 & 6.4591123911 & 2.0257426378 & 20.0318375999 \\
\hline 0 & 8.5081404615 & 0.0899437600 & 10.9015214965 \\
\hline 0 & 9.2300465337 & 4.3094467368 & 13.9580321648 \\
\hline 0 & 9.6127161150 & 4.9910213260 & 17.3523412998 \\
\hline 0 & 9.1124945380 & 0.9429180587 & 20.2902168116 \\
\hline 0 & 7.1194392861 & 2.3855848534 & 10.6511522621 \\
\hline 0 & 6.1299126599 & 3.1839552284 & 14.3389113513 \\
\hline 0 & 9.6164630891 & 2.3419556769 & 12.0805239529 \\
\hline 0 & 9.4781181721 & 3.1258232756 & 9.2919869770 \\
\hline 0 & 6.9138973117 & 5.0149624653 & 18.4187044214 \\
\hline 0 & 6.3859728616 & 0.4842051269 & 15.3887053234 \\
\hline 0 & 6.0484936195 & 0.0667710739 & 12.0078457522 \\
\hline 0 & 7.0707641182 & 4.5497193486 & 8.9794672235 \\
\hline 0 & 8.4197883496 & 2.6737188044 & 18.0783312046 \\
\hline 0 & 8.9213048149 & 1.9010392280 & 15.4198719200 \\
\hline 0 & 0.8342981510 & 7.5539176352 & 17.3916211890 \\
\hline 0 & 1.6637642671 & 7.3456747695 & 20.1124659621 \\
\hline 0 & 3.3630351138 & 5.1390051740 & 11.1533336293 \\
\hline 0 & 4.1445577395 & 9.5099515822 & 13.9320681770 \\
\hline 0 & 4.5675902000 & 10.2338188147 & 17.2635967337 \\
\hline 0 & 3.7661760913 & 5.6263179215 & 20.1693841567 \\
\hline 0 & 1.7931941356 & 7.4172932107 & 10.9856538296 \\
\hline 0 & 1.2961941973 & 8.1955154171 & 14.0253254724 \\
\hline 0 & 4.3918209424 & 7.3966018773 & 12.0822202754 \\
\hline 0 & 4.1246929506 & 7.8199725088 & 9.3189471225 \\
\hline 0 & 1.8955871145 & 9.8127990410 & 18.4096496730 \\
\hline 0 & 1.2223299816 & 5.6044584562 & 15.5850168512 \\
\hline 0 & 0.8497762992 & 5.0947176180 & 12.1341062599 \\
\hline 0 & 1.9276330753 & 9.3292904703 & 8.9677722344 \\
\hline 0 & 3.6900563059 & 7.4713533493 & 18.1025237806 \\
\hline 0 & 4.0153849610 & 7.0748589389 & 15.3708063824 \\
\hline 0 & 6.1408680496 & 7.7056486906 & 17.3135735671 \\
\hline
\end{tabular}




\begin{tabular}{|c|c|c|c|}
\hline 0 & 6.8826992436 & 7.2320571808 & 20.1918474810 \\
\hline 0 & 8.7258979476 & 4.9926973047 & 11.1846930246 \\
\hline 0 & 9.1418861946 & 9.5675968496 & 13.7266359298 \\
\hline 0 & 9.8080947707 & 9.9021129925 & 17.0614841229 \\
\hline 0 & 9.0901338701 & 5.5737929291 & 20.1562538307 \\
\hline 0 & 6.8848898064 & 7.4159391700 & 11.0227580789 \\
\hline 0 & 6.6217377687 & 8.2532448870 & 13.8658109207 \\
\hline 0 & 9.5938228809 & 7.4160650907 & 12.1253376019 \\
\hline 0 & 8.9680675044 & 8.0218035312 & 9.2470801562 \\
\hline 0 & 7.4206823511 & 10.0200493098 & 18.2788263790 \\
\hline 0 & 6.3423541666 & 5.7611290920 & 15.4974975184 \\
\hline 0 & 6.0286544311 & 4.8476029426 & 12.2015817787 \\
\hline 0 & 6.6937210105 & 9.4700478243 & 9.1934689604 \\
\hline 0 & 8.8094622046 & 7.5024893792 & 18.1992353509 \\
\hline 0 & 9.1963416359 & 6.8593555421 & 15.4140343142 \\
\hline $\mathrm{Bi}$ & -0.0768234834 & 1.2646725830 & 10.0815555625 \\
\hline $\mathrm{Bi}$ & 2.6502429225 & 3.6026616333 & 16.4479812524 \\
\hline $\mathrm{Bi}$ & -0.0740246220 & 3.6216765255 & 19.1359232518 \\
\hline $\mathrm{Bi}$ & 2.6802243319 & 1.3056633703 & 12.6573195091 \\
\hline $\mathrm{Bi}$ & 5.2914073048 & 1.1100455342 & 10.0631773084 \\
\hline $\mathrm{Bi}$ & 7.4892551591 & 3.8248720801 & 16.2718508106 \\
\hline $\mathrm{Bi}$ & 5.0594393728 & 3.8088978843 & 19.1813936249 \\
\hline $\mathrm{Bi}$ & 7.7872038879 & 1.2444417657 & 12.9942751082 \\
\hline $\mathrm{Bi}$ & 0.0553554789 & 6.2342564305 & 10.1848708707 \\
\hline $\mathrm{Bi}$ & 2.5771994873 & 8.8289501228 & 16.4528125461 \\
\hline $\mathrm{Bi}$ & 0.0424648856 & 8.8661480944 & 19.2335040589 \\
\hline $\mathrm{Bi}$ & 2.6231517124 & 6.3078487990 & 13.1378599475 \\
\hline $\mathrm{Bi}$ & 5.2516401410 & 6.0828134760 & 10.2474792135 \\
\hline $\mathrm{Bi}$ & 7.8879365911 & 8.7822082668 & 16.1931687072 \\
\hline $\mathrm{Bi}$ & 5.6240082806 & 9.0306595614 & 19.0278561656 \\
\hline $\mathrm{Bi}$ & 7.8779253860 & 6.3226628245 & 13.0166732978 \\
\hline V & -0.0649325516 & 1.2244672782 & 16.1864023305 \\
\hline V & 2.7541936836 & 3.9465483053 & 10.0741723650 \\
\hline V & 0.0165273098 & 3.7418601521 & 12.9710836938 \\
\hline V & 2.5923321783 & 1.1039855959 & 19.2776958411 \\
\hline V & 5.1366148342 & 1.3030692999 & 16.1451909168 \\
\hline V & 8.0405527252 & 3.7733682979 & 10.0241686824 \\
\hline V & 5.0698279644 & 3.7738118026 & 13.1836162909 \\
\hline V & 7.7823229760 & 1.4168468213 & 19.2233050677 \\
\hline V & 0.0335287461 & 6.2801795566 & 16.4849605420 \\
\hline V & 2.8340961845 & 8.5951794892 & 10.0822272328 \\
\hline V & 0.0601516833 & 8.7633131977 & 12.9828496950 \\
\hline V & 2.6349258253 & 6.3472854346 & 19.1364954592 \\
\hline V & 5.0783951554 & 6.3934809105 & 16.4918806439 \\
\hline
\end{tabular}




$\begin{array}{llll}\text { V } & 7.7880159287 & 8.7541650864 & 10.2139010541 \\ \text { V } & 5.2694514667 & 8.7271664929 & 12.9168853581 \\ \text { V } & 7.9197551380 & 6.3256165847 & 19.1735414507 \\ \text { P } & 4.2594175725 & 0.3454270776 & 25.2222301801 \\ \text { P } & 1.6475142361 & 1.9849675613 & 23.2950647472 \\ \text { P } & 0.7005187880 & 2.1757244909 & 25.3145768558 \\ \text { P } & 3.1377520476 & 0.3775916426 & 23.2863769348 \\ \text { P } & 9.4858624978 & 0.5165483362 & 25.4846791197 \\ \text { P } & 6.9346992339 & 2.1319308608 & 23.2719751241 \\ \text { P } & 5.8639008490 & 2.0674518792 & 25.2674782173 \\ \text { P } & 8.4082672607 & 0.3896356267 & 23.3925182182 \\ \text { P } & 4.4851050637 & 3.8063119712 & 24.8907020065 \\ \text { P } & 1.6596855445 & 5.4351511733 & 23.2586688283 \\ \text { P } & 0.6163940202 & 5.3635020483 & 25.2293626192 \\ \text { P } & 3.0551497600 & 3.7901539259 & 23.0436291592 \\ \text { P } & 9.6045387134 & 3.8390486721 & 25.1020219262 \\ \text { P } & 6.9907587099 & 5.3639917312 & 23.1812126647 \\ \text { P } & 5.7603136520 & 5.5260751100 & 25.0052973399 \\ \text { P } & 8.4992185156 & 3.6884192235 & 23.2249672688 \\ \text { P } & 4.3412839482 & 7.2327861650 & 25.1519987102 \\ \text { P } & 1.7291563729 & 8.8100525724 & 23.2653209826 \\ \text { P } & 0.7423216031 & 8.7811886979 & 25.4018988598 \\ \text { P } & 3.2219481356 & 7.1918458309 & 23.1994452257 \\ \text { P } & 9.5442028275 & 7.0869581624 & 25.2749195141 \\ \text { P } & 6.9920811401 & 8.8559707774 & 23.2189651099 \\ \text { P } & 5.9321845983 & 8.8804925693 & 25.1969582418 \\ \text { P } & 8.4733141797 & 7.0511933070 & 23.1599884578\end{array}$

